Letter to the Editor

\title{
Early B cell developmental impairment with progressive B cell deficiency in NFKB2 mutated CVID disease without autoimmunity
}

A R T I C L E I N F O

\section{Keywords:}

NFKB2

B cells

Bone marrow

Autoimmunity

CVID

\begin{abstract}
A B S T R A C T
This study provides evidence for a novel role for NFKB2 in human B cell development in the bone marrow and in the periphery, leading to progressive peripheral B cell deficiency not always combined with autoimmune phenomena, broadening thus the clinical spectrum of NFKB2 mutated CVID disease and implying an essential role for NFKB2 in early human B cell development.
\end{abstract}

To The Editor:

NFKB2 is the cornerstone of the non-canonical NF-kB pathway, which has been shown to be critical for the development of secondary lymphoid organs, B cell development, and the humoral response to Tdependent and T-independent antigens [1,2]. Monoallelic loss-offunction mutations in NFKB2 were identified in a limited number of patients with a CVID-like disease characterized by early-onset infections, hypogammaglobulinemia, variable B cell lymphopenia, and impaired terminal B cell development [4-8]. Additional features of this disease found in some, but not all patients, include reduced NK cell numbers, ACTH deficiency, alopecia, and trachyonichia [3]. In contrast, two heterozygous gain of function mutations in NFKB2 leading to nuclear retention and constitutive NF-kB activation have been associated with a combined immunodeficiency characterized by B cell lymphopenia and variable $\mathrm{T}$ cell lymphopenia [8]. While peripheral B cell lymphopenia has been reported in patients with NFKB2 mutations $[4,5,7,8]$, early B cell development has not been previously studied in these patients. We present a patient with a frameshift mutation in NFKB2 and document an early B cell developmental impairment in the bone marrow with progressive peripheral B cell deficiency.

The index patient is the son of non-consanguineous Italian parents. He had a history of recurrent upper respiratory tract infections and measles complicated by pneumonia that required hospitalization at the age of 5 years. He was initially treated at an outside medical center and came to our attention at 14 years of age. His immunologic evaluation was notable for reduced immunoglobulins and absent humoral response to vaccinations, despite normal numbers of $\mathrm{T}, \mathrm{B}$, and NK cells (Supplemental Table 1). He was treated with immunoglobulin replacement therapy, which led to significant reduction of infectious episodes. Lung CT scan did not evidence any pathologic findings, while sinus CT scan showed mucosal thickening at the maxillary sinus, compatible with chronic sinusitits (Supplemental Fig. 1A and B respectively). Annual abdomen ultrasonographies revealed lack of splenomegaly and lymphoadenopathies. He developed a progressive reduction in the numbers of his circulating B cells, with a concomitant increase in $\mathrm{T}$ cells (Supplemental Table 1 and Fig. 1A) in the absence of leukopenia or lymphopenia (Fig. 1A). Bone marrow evaluation revealed an early $\mathrm{B}$ cell developmental arrest at the $\mathrm{CD} 34^{+} \mathrm{CD} 22^{+} \mathrm{CD} 19^{+} \mathrm{CD} 10^{\mathrm{hi}} \mathrm{CD} 45^{\text {lo }}$ pre-BI stage (Fig. 1B), similar to that found in a patient with $\operatorname{Ig} \beta$ deficiency (Fig. 1C). Next-generation sequencing of 264 genes associated with primary immunodeficiency identified a heterozygous mutation NFKB2 (c.2257C > T; p.Arg853*) (Fig. 1D). This mutation, which has been previously reported, inhibits p100 processing and nuclear translocation of p52 [3]. Compared to previously published patients with this mutation, our proband, who is currently 40 years old, has had no manifestations of autoimmune disease (Supplemental Table 2).

The role of NFKB2 in B cell development has been mainly studied in mice [2,9]. NFKB2 knock-out mice showed a reduction in the B cell compartment both in the spleen and in the bone marrow, which was not apoptosis-related [2]. T-dependent and-independent immune responses were severely compromised in the knock-out animal model, with associated impaired germinal center formation [2]. The index patient had a block at the pre-BI stage, indicating an important role for NFKB2 in human early B cell development, as also suggested by the animal models $[2,9]$, associated with a progressive reduction in peripheral transitional B cells (Supplemental Table 3).

Monoallelic NFKB2 mutations have been associated with variable clinical presentation in a small subset of patients with primary immunodeficiencies [2-8]. The p.Arg853* mutation has been reported in 9 patients so far [6] (Supplemental Table 2). Patients with this mutation have most of the originally described features of the disease: hypogammaglobulinemia, respiratory infections, ACTH deficiency, hypothyroiditis, alopecia universalis, and skin disease (Supplemental Table 2). Of note, the index patient presented with hypogammaglobulinemia and only mild respiratory infections [6] (Supplemental Table 2), suggesting that the same NFKB2 mutation may give rise to a wide spectrum of clinical presentation.

Taken together, our data provide evidence for a novel role for NFkB2 in early human B cell development. In addition, our data suggest that the clinical presentation of patients carrying the same NFKB2 mutation (p.Arg853*) can be variable, with even absence of major hallmarks of the disease, and thus, next generation sequencing techniques may be helpful in identifying affected patients.

Supplementary data to this article can be found online at 
A.
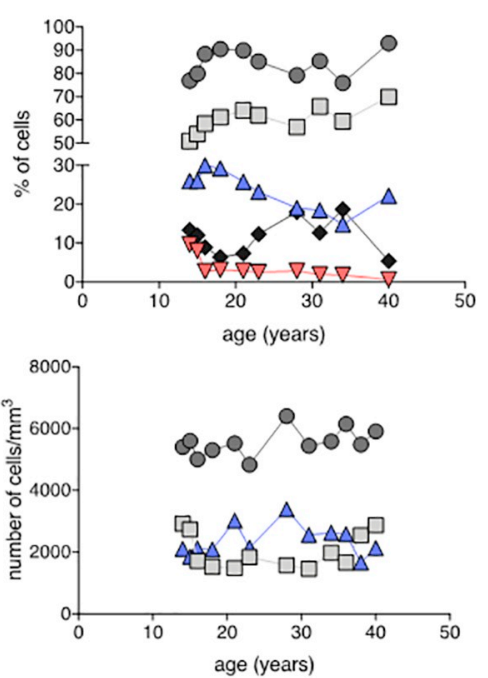

- WBCs

$\square$ ALCs

$\triangle$ ANCs

$\square \mathrm{CD} 4$

$\triangle \mathrm{CD} 8$

$\nabla$ CD19

- CD56

C.

\section{B cell precursors}

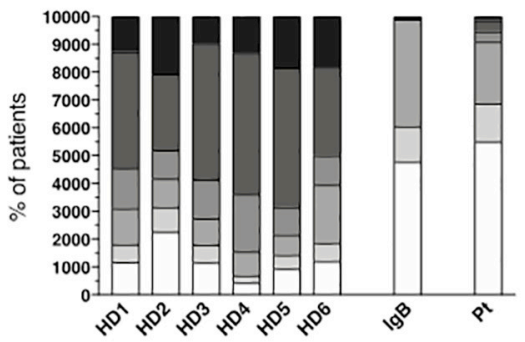

Pro B

Pre BI

$\square$ Pre Blla

$\square$ Pre Bllb

Imm
B

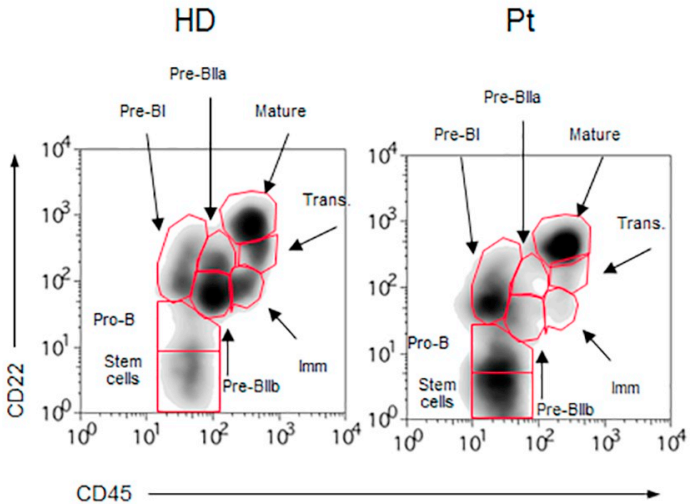

c. $2557 \mathrm{c}>\mathrm{T}\left(\mathrm{p} . \operatorname{Arg} 853^{\star}\right)$

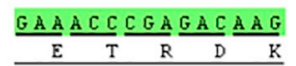

$\ldots \ldots \ldots$

GAAACCCGAGACAAG

$\square$ Stem Cells
HD

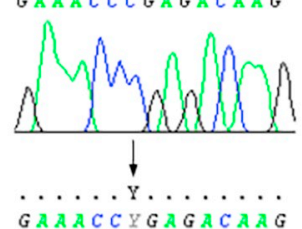

Pt

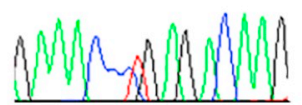

Fig. 1. Immunological evaluation of the NFKB2 mutated patient. A. Lymphocyte subset evaluation during 26 year follow-up (upper panel) and differential blood count values during 26 year follow-up (lower panel). B. B cell development in the bone marrow from a healthy donor (HD: left panel) and the index patient (Pt: right panel). C. Comparison of the early B cell developmental pattern in the bone marrow from healthy controls (HDs; $n=6$ ), a patient affected with agammaglobulinemia due to Igbeta deficiency (IgB) and the index patient (Pt). D. Electropherograms showing the heterozygous c.2557C > T variant in NFKB2 (HD: upper panel; index patient (Pt): lower panel).

https://doi.org/10.1016/j.clim.2018.11.014.

\section{Acknowledgments}

We would like to thank the patients, the patients' families and the nurses for all their efforts. We thank Raif S. Geha, Janet Chou, and the members of their laboratories for providing the next-generation DNA sequencing data and for their discussions of this work.

\section{Funding}

The research leading to these results has received funding from the European Community's Seventh Framework Programme FP7/ 2007-2013 under grant agreement no 201549 (EURO-PADnet HEALTH-F2-2008-201549) and from the Italian Ministerial Grant GR2010-2315762. The research leading to these results also received funding from the "Fondazione C. Golgi", Brescia, Italy and the Jeffrey Modell Foundation.

\section{Disclosure of conflicts of interest}

The authors declare no conflict of interest.

\section{References}

[1] S. Gerondakis, R. Grumont, R. Gugasyan, L. Wong, I. Isomura, W. Ho, A. Banerjee, Unravelling the complexities of the NF-kappaB signalling pathway using mouse knockout and transgenic models, Oncogene 25 (51) (2006 Oct 30) 6781-6799.

[2] J.H. Caamaño, C.A. Rizzo, S.K. Durham, D.S. Barton, C. Raventós-Suárez C.M. Snapper, Bravo. RNuclear factor (NF)-kappa B2 (p100/p52) is required for normal splenic microarchitecture and B cell-mediated immune responses, J. Exp. Med. 187 (2) (1998 Jan 19) 185-196.

[3] K. Chen, E.M. Coonrod, A. Kumánovics, Z.F. Franks, J.D. Durtschi, R.L. Margraf, W. Wu, N.M. Heikal, N.H. Augustine, P.G. Ridge, H.R. Hill, L.B. Jorde, A.S. Weyrich, G.A. Zimmerman, A.V. Gundlapalli, J.F. Bohnsack, K.V. Voelkerding, Germline mutations in NFKB2 implicate the noncanonical NF-kB pathway in the pathogenesis of common variable immunodeficiency, Am. J. Hum. Genet. 93 (5) (2013 Nov 7) 812-824.

[4] A.W. Lindsley, Y. Qian, C.A. Valencia, K. Shah, K. Zhang, A. Assa'ad, Combined immune deficiency in a patient with a novel NFKB2 mutation, J. Clin. Immunol. 34 (8) (2014 Nov) 910-915, https://doi.org/10.1007/s10875-014-0095-3.

[5] C.E. Lee, D.A. Fulcher, B. Whittle, R. Chand, N. Fewings, M. Field, D. Andrews, C.C. Goodnow, M.C. Cook, Autosomal-dominant B-cell deficiency with alopecia due to a mutation in NFKB2 that results in nonprocessable p100, Blood 124 (19) (2014 Nov 6) 2964-2972, https://doi.org/10.1182/blood-2014-06-578542.

[6] V. Lougaris, G. Tabellini, M. Vitali, M. Baronio, O. Patrizi, G. Tampella, A. Biasini, D. Moratto, S. Parolini, A. Plebani, Defective natural killer-cell cytotoxic activity in NFKB2-mutated CVID-like disease, J. Allergy Clin. Immunol. 135 (6) (2015 Jun) 1641-1643, https://doi.org/10.1016/j.jaci.2014.11.038.

[7] C. Shi, F. Wang, A. Tong, X.Q. Zhang, H.M. Song, Z.Y. Liu, W. Lyu, Y.H. Liu, W.B. Xia, NFKB2 mutation in common variable immunodeficiency and isolated adrenocorticotropic hormone deficiency: a case report and review of literature, Medicine (Baltimore) 95 (40) (2016 Oct) e5081(Review).

[8] H.S. Kuehn, J.E. Niemela, K. Sreedhara, J.L. Stoddard, J. Grossman, C.A. Wysocki, M.T. de la Morena, M. Garofalo, J. Inlora, M.P. Snyder, D.B. Lewis, C.A. Stratakis, T.A. Fleisher, S.D. Rosenzweig, Novel nonsense gain-of-function NFKB2 mutations associated with a combined immunodeficiency phenotype, Blood 130 (13) (2017 Sep 28) 1553-1564, https://doi.org/10.1182/blood-2017-05-782177.

[9] E. Claudio, S. Saret, H. Wang, U. Siebenlist, Cell-autonomous role for NF-kappa B in immature bone marrow B cells, J. Immunol. 182 (6) (2009 Mar 15) 3406-3413. 
Vassilios Lougaris $^{\mathrm{a}, *}$, Daniele Moratto ${ }^{\mathrm{b}}$, Manuela Baronio ${ }^{\mathrm{a}}$, Tiziana Lorenzini ${ }^{\mathrm{a}}$, Stefano Rossi ${ }^{\mathrm{a}}$, Luisa Gazzurelli ${ }^{\mathrm{a}}$, Maria Pia Bondioni ${ }^{\mathrm{c}}$, Alessandro Plebani ${ }^{\mathrm{a}}$ ${ }^{a}$ Pediatrics Clinic and Institute for Molecular Medicine A. Nocivelli, Department of Clinical and Experimental Sciences, University of Brescia, Spedali Civili di Brescia, Italy
${ }^{\mathrm{b}}$ Institute for Molecular Medicine A. Nocivelli, Department of Pathology, Laboratory of Genetic Disorders of Childhood, Department of Molecular and Translational Medicine, University of Brescia, Spedali Civili di Brescia, Italy ${ }^{\mathrm{c}}$ Pediatric Radiology, University of Brescia and ASST Spedali Civili di Brescia, Brescia, Italy E-mail address: vlougarisbs@yahoo.com (V. Lougaris).

*Corresponding author: Pediatrics Clinic and Institute of Molecular Medicine "A. Nocivelli”, Department of Clinical and Experimental Sciences, University of Brescia, Piazzale Spedali Civili 1, 25123, Brescia, Italy. 